\title{
Proposal of a consistent framework to integrate geothermal potential classification with energy extraction
}

\author{
G. Falcone \\ Department of Geothermal Engineering and Integrated Energy Systems, Institute of Petroleum Engineering, \\ Clausthal University of Technology, Clausthal-Zellerfeld, Germany
}

Correspondence to: G. Falcone (gioia.falcone@ @u-clausthal.de)

Received: 24 June 2014 - Revised: 15 February 2015 - Accepted: 24 February 2015 - Published: 12 March 2015

\begin{abstract}
The classification of geothermal resources is dependent on the estimate of their corresponding geothermal potential, so adopting a common assessment methodology would greatly benefit operators, investors, government regulators and consumers.

Several geothermal classification schemes have been proposed, but, to date, no universally recognised standard exists. This is due to the difficulty in standardising fundamentally different geothermal source and product types. The situation is not helped by the accepted use of inconsistent jargon among the geothermal community. In fact, the term "geothermal potential" is often interpreted differently by different geothermal practitioners.

This paper highlights the importance of integrating the classification of geothermal potential with that of geothermal energy extraction from well-defined development projects. A structured progression, from estimates of in situ quantities for a given prospect to actual production, is needed. Employing a unique, unambiguous framework would ensure that the same resource cannot exist simultaneously under different levels of maturity of the estimate (as in double bookings of resources), which would let stakeholders better assess the level of risk involved and the steps needed for a geothermal potential to achieve commercial extraction.
\end{abstract}

\section{Introduction}

There is a real challenge to reconcile nomenclature for in situ or in-place quantities of geothermal energy, potential (technical or economic or sustainable or developable), resources (inferred or indicated or measured) and reserves (probable or proven), to name but a few.

According to Rybach (2010), the theoretical potential describes the physically usable energy supply (heat in place). Due to technical, structural and administrative limitations only small fractions of the theoretical potential can actually be used. The technical potential describes the fraction of the theoretical potential that can be used under the existing technical restrictions (currently available technology). The economic potential describes the time- and location-dependent fraction of the technical potential that can be economically utilised within the energy system under consideration. The sustainable potential is a fraction of the economic potential; it describes the fraction that can be utilised by applying sustainable production levels. The developable potential describes the fraction of the sustainable potential that can be developed under realistic conditions (regulations, environmental restrictions).

Often, the generic term "potential" is used in the public domain, without clear indication of what particular type of potential is being referred to (theoretical, technical, economic, sustainable or developable). Inevitably, this generates confusion among the stakeholders as to the actual expectations from a given geothermal prospect or development.

Classifying geothermal resources on the basis of the theoretical potential (or heat in place) leads to large figures that can be misleading and may be wrongly interpreted as recoverable energy. However, when the process to be implemented in order to recover a given resource is uncertain, the theoretical potential may represent the only estimate on hand.

Different methods are used to estimate geothermal potential, including power density (usually expressed in terms of MW km${ }^{-2}$ ), stored heat (or volume method, independent of the method or rate of heat extraction) and numerical reservoir 

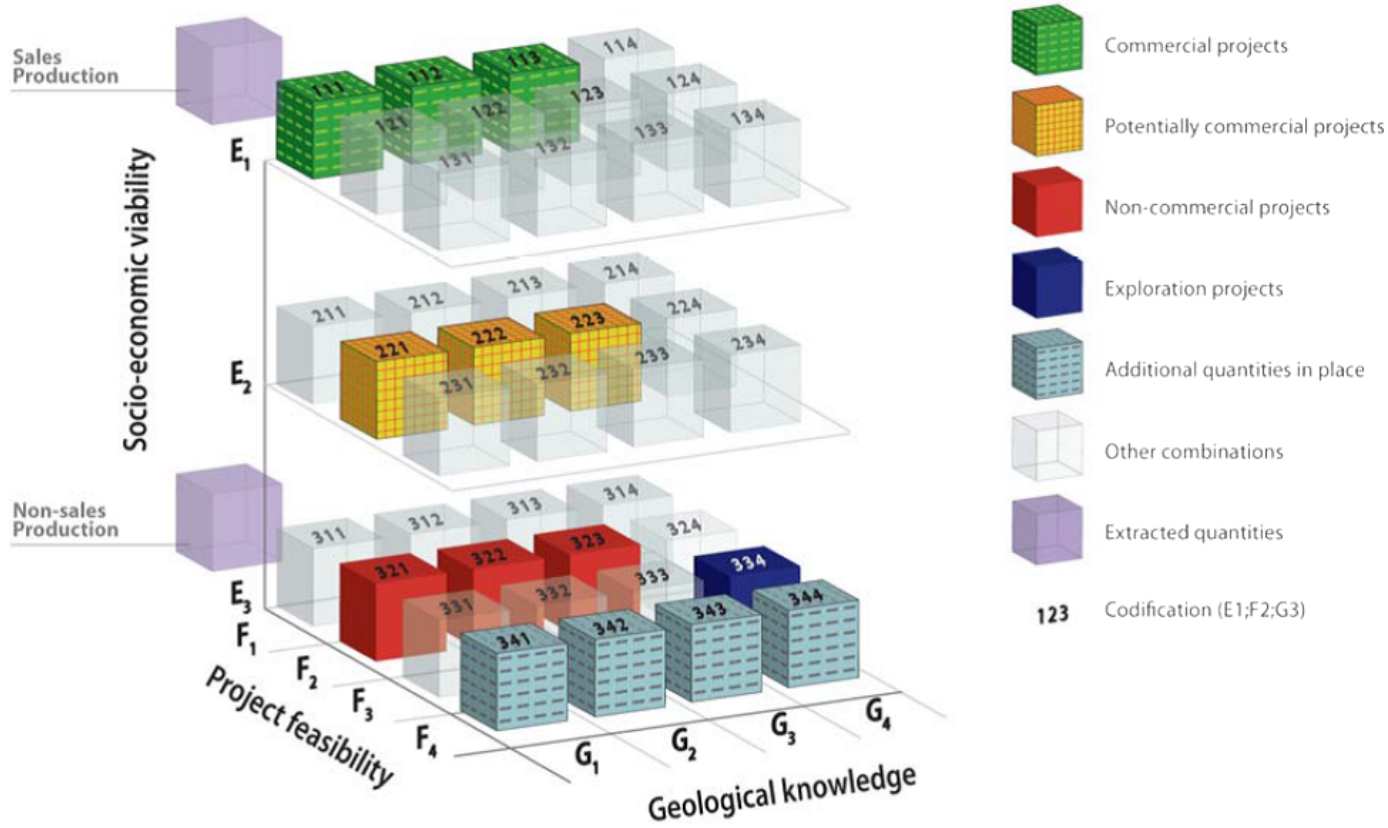

Figure 1. UNFC-2009 categories and examples of classes (ECE, 2013).

modelling (generally not suitable in the exploration and early development stage of a project). The resulting estimates carry different levels of confidence depending on whether they are based on indirect or direct evidence (e.g. geological studies and surface exploration vs. well logging and flow testing) and on the level of field maturity (exploration, appraisal or development). Inevitably, broad estimates of geothermal potential at country, continent or world scale rely on the assumption of parameters that are unknown.

Given the current global trend of increasing energy demand and the constant dilemma of whether renewable energy resources will be able to supplement or even replace conventional energy resources, it is crucial to assess future geothermal supplies at a large scale. This translates into a focus today on estimating and classifying geothermal theoretical potential, i.e. additional quantities in place that could be converted into actual production in the foreseeable future.

Unconventional, undiscovered and/or currently unrecoverable geothermal resources therefore need to be captured vis-à-vis the conventional, discovered and/or recoverable resources, in a consistent and structured way, so as to help stakeholders understand the level of risk involved and the steps needed for a geothermal potential to achieve commercial extraction.

\section{The UNFC-2009}

Several geothermal resources classification schemes have been proposed to date, yet no universally recognised standard exists. Falcone et al. (2013) provided a critical review of key approaches proposed to date for geothermal resources classification.

The United Nations Framework Classification for Fossil Energy and Mineral Reserves and Resources 2009 (UNFC2009) differs from other systems as it classifies estimated resource quantities using three axes: socio-economic variability $(E)$, project feasibility $(F)$ and geological knowledge $(G)$. The first set of categories (the $E$ axis) designates the degree of favourability of social and economic conditions in establishing the commercial viability of the project, including consideration of market prices and relevant legal, regulatory, environmental and contractual conditions. The second set (the $F$ axis) designates the maturity of studies and commitments necessary to implement mining plans or development projects. These extend from early exploration efforts before a deposit or accumulation has been confirmed to exist through to a project that is extracting and selling a commodity; they reflect standard value chain management principles. The third set of categories (the $G$ axis) designates the level of confidence in the geological knowledge and potential recoverability of the quantities (ECE, 2013). Combinations of these criteria create a three-dimensional system as shown in Fig. 1.

The UNFC-2009 can already be used to normalise the classification of hydrocarbon and mineral resources. It also ensures alignment with widely used systems such as the Committee for Mineral Reserves International Reporting Standards (CRIRSCO) Intenational Reporting Template (IRT) and the Society of Petroleum Engineers (SPE)/World Petroleum Council (WPC)/American Association of Petroleum Geologists (AAPG)/Society of Petroleum 


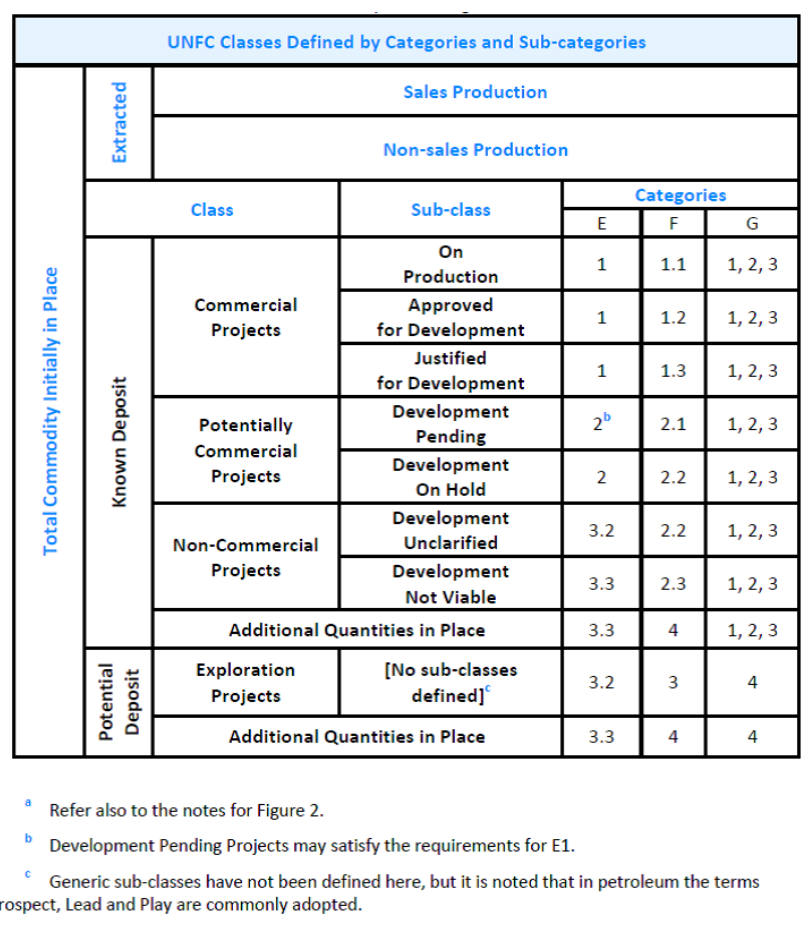

Figure 2. UNFC-2009 classes and sub-classes defined by subcategories $^{\mathrm{a}}$ (ECE, 2013). For the definition of categories and subcategories and supporting explanations, see Annex I and Annex II of ECE (2013).

Evaluation Engineers (SPEE) Petroleum Resource Management System (PRMS).

The United Nations Economic Commission for Europe (UNECE, 2012) called upon its Expert Group on Resource Classification (EGRC) to develop ideas on how the UNFC2009 could apply to and integrate renewable energy resources. Following agreement at the fourth session of the UNECE EGRC held in Geneva, April 2013, a Task Force on the Application of UNFC-2009 to Renewable Energy Resources was established. The Task Force (2014) recently released specifications for the application to renewable energy resources for public comment. The aim is that of complementing these specifications with commodity-specific specifications for each type of renewable energy source, including geothermal.

In September 2014, the International Geothermal Association (IGA) and the UNECE signed a Memorandum of Understanding (MoU) to develop a globally applicable harmonised standard for reporting geothermal resources. Such a standard will ensure greater consistency and transparency in financial reporting and enhance management of geothermal resources. Under this MoU, the IGA will work towards providing technology-specific rules ("specifications") for the application of the UNFC-2009 to geothermal resources. This work will be overseen by the UNECE EGRC. The geothermal specifications will provide the foundation and guidelines for consistent application of UNFC-2009 for geothermal resources, as well as a meaningful comparison of geothermal resource estimates with other energy resources.

Below there is a discussion on how UNFC-2009 could apply to geothermal energy resources, with a focus on the classification of potential future recovery by successful exploration activities and additional quantities in place associated with known and potential deposits, using the same framework that also allows classifying economic projects.

\subsection{Potential vs. known deposit in UNFC-2009}

With reference to Fig. 2, the UNFC-2009 allows differenciating between a known deposit and a potential deposit. A known deposit is a deposit that has been demonstrated to exist by direct evidence; a potential deposit is a deposit that has not yet been demonstrated to exist by direct evidence (e.g. drilling and/or sampling) but is assessed as potentially existing based primarily on indirect evidence (e.g. surface or airborne geophysical measurements) (ECE, 2013). Within UNFC-2009, the geologic uncertainty for discovered quantities is described using categories G1 to G3, while the geologic uncertainty for undiscovered quantities is described using category G4. Thus, a potential deposit includes quantities classified on the $G$ axis as G4: "estimated quantities associated with a potential deposit, based primarily on indirect evidence" (ECE, 2013).

\subsection{Additional quantities in place in UNFC-2009}

With reference to Fig. 2, the UNFC-2009 allows reporting additional quantities in place as both quantities associated with a known deposit that will not be recovered by any currently defined development project or mining operation and quantities associated with a potential deposit that would not be expected to be recovered even if the deposit is confirmed (ECE, 2013). Additional quantities are classified on the $F$ axis as F4 when "no development project or mining operation has been identified/in situ (in-place) quantities that will not be extracted by any currently defined development project or mining operation" (ECE, 2013). F4 means that no recovery factor is superimposed to estimates of in situ quantities. Additional quantities in place can be sub-classified as follows on the basis of the current state of technological developments (ECE, 2013):

a. "F4.1: the technology necessary to recover some or all of the these quantities is currently under active development, following successful pilot studies on other deposits, but has yet to be demonstrated to be technically feasible for the style and nature of deposit in which that commodity or product type is located;

b. F4.2: the technology necessary to recover some or all of the these quantities is currently being researched, but no successful pilot studies have yet been completed; 


\begin{tabular}{|c|c|c|c|c|}
\hline & & Low Estimate & Best Estimate & High Estimate \\
\hline \multirow{3}{*}{ 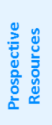 } & Prospect & E3.2,F3.1,G4.1 & E3.2,F3.1,G4.1+G4.2 & $\mathrm{E} 3.2, \mathrm{~F} 3.1, \mathrm{G} 4.1+\mathrm{G} 4.2+\mathrm{G} 4.3$ \\
\hline & Lead & $\mathrm{E} 3.2, \mathrm{~F} 3.2, \mathrm{G} 4.1$ & $\mathrm{E} 3.2, \mathrm{~F} 3.2, \mathrm{G} 4.1+\mathrm{G} 4.2$ & $\mathrm{E} 3.2, \mathrm{~F} 3.2, \mathrm{G} 4.1+\mathrm{G} 4.2+\mathrm{G} 4.3$ \\
\hline & Play & E3.2,F3.3,G4.1 & $\mathrm{E} 3.2, \mathrm{~F} 3.3, \mathrm{G} 4.1+\mathrm{G} 4.2$ & $\mathrm{E} 3.2, \mathrm{~F} 3.3, \mathrm{G} 4.1+\mathrm{G} 4.2+\mathrm{G} 4.3$ \\
\hline
\end{tabular}

Figure 3. Mapping of UNFC-2009 exploration projects to PRMS prospective resources (ECE, 2013). For the definitions of prospect, lead and play, see PRMS (SPE et al., 2007). For the definition of categories and sub-categories and supporting explanations, see Annex I and Annex II of ECE (2013). "Low", "best" and "high" reflect the degree of uncertainty associated with the estimates. A lowestimate scenario is directly equivalent to a high confidence estimate, whereas a best-estimate scenario is equivalent to the combination of the high confidence and moderate confidence estimates. A high-estimate scenario is equivalent to the combination of high, moderate and low confidence estimates. Quantities may be estimated using deterministic or probabilistic methods.

c. F4.3: the technology necessary to recover some or all of these quantities is not currently under research or development."

\subsection{Exploration projects in UNFC-2009}

With reference to Fig. 2, in the UNFC-2009 exploration projects are classified on the $G$ axis as $\mathrm{G} 4$ and on the $F$ axis as F3: "feasibility of extraction by a defined development project or mining operation cannot be evaluated due to limited technical data", and "very preliminary studies (e.g. during the exploration phase), which may be based on a defined (at least in conceptual terms) development project or mining operation, indicate the need for further data acquisition in order to confirm the existence of a deposit in such form, quality and quantity that the feasibility of extraction can be evaluated" (ECE, 2013).

\subsection{Combining $E, F$ and $G$ for exploration projects and additional quantities in place in UNFC-2009}

Exploration projects and additional quantities in place are classified on the $E$ axis as E3.

When combining the $E, F$ and $G$ categories, an exploration project in UNFC-2009 would fall under E3 F3 G4, and additional quantities in place would fall under E3 F4 G1, 2, 3 (for known deposits) or E3 F4 G4 (for unknown deposits). E3 implies "extraction and sale is not expected to become economically viable in the foreseeable future or evaluation is at too early a stage to determine economic viability" (ECE, 2013).

Considering that geothermal systems, like petroleum systems, deal with continuous fluid flow, the current bridging document between the PRMS (SPE et al., 2007) and UNFC-2009 could be used to map geothermal exploration projects and additional quantities in place to UNFC-2009.

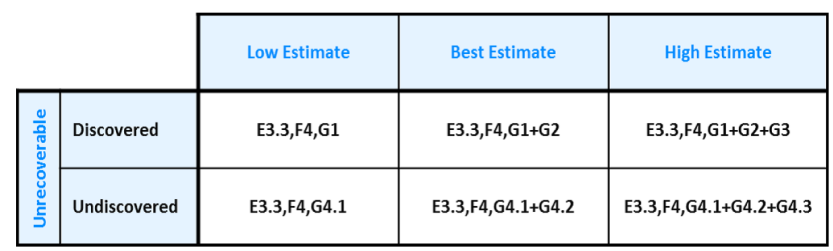

Figure 4. Mapping of UNFC-2009 additional quantities in place to PRMS unrecoverable quantities (ECE, 2013). "Unrecoverable" implies that the technology has not been demonstrated to be commercially viable and is not currently under active development, and/or there is not yet any direct evidence to indicate that it may reasonably be expected to be available for commercial application within 5 years. For the definition of categories and sub-categories and supporting explanations, see Annex I and Annex II of ECE (2013). "Low", "best" and "high" reflect the degree of uncertainty associated with the estimates. A low-estimate scenario is directly equivalent to a high confidence estimate, whereas a best-estimate scenario is equivalent to the combination of the high confidence and moderate confidence estimates. A high-estimate scenario is equivalent to the combination of high, moderate and low confidence estimates. Quantities may be estimated using deterministic or probabilistic methods.

There are four cells within the $E-F$ matrix that map directly and uniquely to corresponding PRMS project maturity classes; these cells relate to exploration projects (prospective resources in PRMS) and additional quantities in place (unrecoverable in PRMS) (ECE, 2013). Accordingly, geothermal exploration projects could be further defined via subcategories as E3.2 F3.1,2,3 G4, where the F3 sub-categories allow specifying the level of maturity of the project (Fig. 3). Similarly, additional quantities in place could be defined as E3.3 F4.1, 2, 3 G4 (Fig. 4), with the F4 sub-categories indicating the current state of technological developments.

E3.2 indicates that "economic viability of extraction cannot yet be determined due to insufficient information (e.g. during the exploration phase)" (ECE, 2013), while E3.3 suggests that, "on the basis of realistic assumptions of future market conditions, it is currently considered that there are not reasonable prospects for economic extraction and sale in the foreseeable future" (ECE, 2013).

Considering that, for recoverable estimates of energy resources that are extracted as fluids, their mobile nature generally precludes assigning recoverable quantities to discrete parts of an accumulation, recoverable quantities should be evaluated on the basis of the impact of the development scheme on the accumulation as a whole and are usually categorised on the basis of three scenarios or outcomes that are equivalent to G1, G1+G2 and G1+G2+G3 (ECE, 2013). $\mathrm{G} 1, \mathrm{G} 1+\mathrm{G} 2$ and $\mathrm{G} 1+\mathrm{G} 2+\mathrm{G} 3$ represent single specific scenarios that are representative of the extent of the range of uncertainty of the recoverable/potentially recoverable quantities and they correspond to low estimates (high confidence), 
best estimates (high and moderate confidence) and high estimates (high, moderate and low confidence), respectively.

\section{Conclusions}

The threefold UNFC-2009 framework allows one to capture the nuances of each geothermal quantity, from in situ potential to confirmed economic production. Its flexibility and granularity permits the classification of quantities that have been estimated primarily on the basis of indirect evidence (e.g. country-based resource mapping studies), as well as quantities at a more specific project level.

UNFC-2009 deliberately avoids the use of ambiguous jargon, having benefited from valuable lessons learnt from the fossil fuels and solid mineral sectors. Nevertheless, the development of dedicated geothermal specifications is needed to make UNFC-2009 fully functional for geothermal resources classification, as it was originally developed for non-renewable energy.

Edited by: G. Beardsmore

Reviewed by: P. Dobson and one anonymous referee

\section{References}

ECE - Economic Commission for Europe: United Nations Framework Classification for Fossil Energy and Mineral Reserves and Resources 2009 incorporating Specifications for its Application, United Nations Publication, ECE Energy Series No.42, ISBN 978-92-1-117073-3, 2013.

Falcone, G., Gnoni, A., Harrison, B., and Alimonti, C.: Classification and Reporting Requirements for Geothermal Resources, European Geothermal Congress 2013, Pisa, Italy, 3-7 June 2013.

Rybach, L.: The Future of Geothermal Energy, Proceedings of World Geothermal Congress, Bali, Indonesia, 25-30 April 2010.

Society of Petroleum Engineers (SPE), American Association of Petroleum Geologists (AAPG), World Petroleum Council (WPC), Society of Petroleum Evaluation Engineers (SPEE): Petroleum Resources Management System, available at: http://www.spe.org/industry/docs/Petroleum_Resources_ Management_System_2007.pdf, downloaded on 26 May 2014.

Task Force on the Application of UNFC-2009: Draft Specifications for Application of UNFC-2009 to Renewable Energy Resources, presented for public comment on 12 June 2014, available at: http://www.unece.org/fileadmin/DAM/energy/se/pdfs/UNFC/ unfc2009_RE_Specs_publcomm_14/RE_Specs_12.06.2014. pdf, downloaded on 16 June 2014.

UNECE: United Nations Economic Commission for Europe Energy industry calls for UNFC to embrace renewables, press release, 5 November 2012. 